Disponível em:

http://editora.unoesc.edu.br/index.php/race

Race, Joaçaba, v. 14, n. 2, p. 451-478, maio/ago. 2015

\title{
APLICABILIDADE DA ESCALA ECCB AO PARADIGMA DA MODELAGEM DE SEGUNDA ORDEM: UMA ANÁLISE DO CONSUMO CONSCIENTE DE COSMÉTICOS
}

\section{Applicability of the ECCB Scale to the Second Order Modeling Paradigm: an analysis of cosmetic conscious consumption}

\begin{abstract}
Wagner Junior Ladeira
E-mail:wjladeira@gmail.com

Doutor em Gestão de Tecnologia e Inovação pela Universidade Federal do Rio Grande do Sul; Mestre em Administração pela Universidade Federal do Rio Grande do Sul; Professor da Universidade do Vale do Rio dos Sinos; Professor de Pós-graduação do Centro Universitário La Salle e da Faculdade de Tecnologia Senac, RS. Endereço pra contato: Avenida Unisinos, 950, Cristo Rei, 93022-000, São Leopoldo, Rio Grande do Sul, Brasil.
\end{abstract}

Fernando Oliveira Santini

E-mail: santiniconsultores@terra.com.br Doutor em Administração pela Pontifícia Universidade Católica do Rio Grande do Sul; Mestre em Administração e Negócios pela Pontifícia Universidade Católica do Rio Grande do Sul; Professor da Escola Superior de Propaganda e Marketing e da Universidade do Vale do Rio dos Sinos.

Clecio Falcão Araujo

E-mail: clecioa@bol.com.br

Doutor e Mestre em Administração e Negócios com ênfase em Marketing pela Pontifícia Universidade Católica do Rio Grande do Sul; Professor de pesquisa de Marketing da Faculdade Senac, RS; Pesquisador no Núcleo de Apoio à Gestão da Inovação.

Artigo recebido em 08 de setembro de 2014. Aceito em 30 de abril de 2015. 
Resumo

No presente artigo teve-se como objetivo analisar as influências dos constructos de primeira e segunda ordem da escala Ecologically Conscious Consumer Behavior (ECCB) no consumo consciente de cosméticos. Para alcançar esse objetivo, foi realizada uma survey com 554 consumidores de três marcas de cosméticos na grande Porto Alegre. Os resultados apontaram a existência de diferentes relações entre os constructos nos quatro modelos hierárquicos estudados, contrastando com estudos publicados na área. O modelo mais parcimonioso demonstrou ausência de relação na percepção da eficácia, coeficientes mais fortes no liberalismo e moderados no altruísmo e na preocupação ambiental, diferente dos estudos das escalas originais. O conjunto de resultados propõe uma forma de análise de Modelagem de Equações Estruturais (MEE) pouco utilizada no meio acadêmico, demonstrando, assim, vantagens de se utilizar a modelagem de segunda ordem em estudo de modelagem de equações estruturais. Por fim, realizou-se uma análise crítica do uso da MEE de segunda ordem e propuseram-se novas análises para superar as limitações deste estudo.

Palavras-chave: Ecologically Conscious Consumer Behavior. Modelagem de equações estruturais. Modelagem de segunda ordem.

\title{
Applicability of the ECCB Scale to the Second Order Modeling Paradigm: an analysis of cosmetic conscious consumption
}

\begin{abstract}
This article aimed to analyze the influences of the constructs of first and second order of the Ecologically Conscious Consumer Behavior (ECCB) scale in conscious cosmetics consumption. To achieve the objective of this article, a survey of 554 consumers in three major cosmetic brands in big Jolly Harbour was held. The results indicate the existence of different relationships among the constructs in the four hierarchical models studied, in contrast to studies published in the area. The most parsimonious model showed no relationship in the perception of efficiency, stronger coefficients in liberalism and moderate in altruism and in environmental concern, different from the original scales studies. The set of results suggests a form of MEE analysis little used in academia, thereby, demonstrating the advantages of using modeling in the study of second-order structural equation modeling. Finally, we carried out a critical analysis of the use of MEE second order and new analyzes have been proposed to overcome the limitations of this study.

Keywords: Ecologically Conscious Consumer Behavior. Structural equation modeling. Modeling second order.
\end{abstract}

\section{INTRODUÇÃO}

Substâncias como formaldeído e o ácido glioxílico são historicamente usadas na indústria de cosméticos. Em razão da emissão de cheiros e sabores desagradáveis, várias empresas produtoras de cosméticos procuram inibir as sensações fortes dessas substâncias com o uso de matérias-primas ecologicamente corretas. Recentemente, essas empresas 
estão sofrendo pressão de consumidores e órgãos regulamentadores (como a Agência Nacional de Vigilância Sanitária (Anvisa)) para diminuir o uso dessas substâncias. Esse fato se deve à consciência ambiental dos consumidores, que vem modificando a forma como as empresas investem seus recursos (HAANÄÄ, 2007; PAÇO; RAPOSO, 2010).

Procurando respeitar a consciência ambiental do consumidor, empresas como De Sirius Cosméticos, Itallian Color, Inoar, Natura e Boticário têm utilizado essências naturalmente brasileiras, investindo cada vez mais no apelo sustentável de seus cosméticos, mesmo que os produtos não sejam 100\% orgânicos. É o exemplo dos óleos de Argan, Dendê, Castanhas da Amazônia e Murumuru que são utilizados em diversos cremes e xampus de tratamento para a pele e os cabelos.

Esses investimentos feitos por empresas do setor de cosméticos mostram a importância da corrente verde na comercialização de produtos. Estudos em marketing já abordam a preocupação com a consciência ambiental há algumas décadas (MITCHELL, 1983; SAMDAHL; ROBERTSON, 1989; ROBERTS, 1996; STRAUGHAN; ROBERTS, 1999; PAÇO; RAPOSO, 2010), entretanto, restam perguntas não sanadas pela academia, como, por exemplo: Será que a percepção da eficácia de um produto pelo consumidor irá influenciar o consumo ecologicamente consciente? Será que o grau de liberalismo do consumidor pode demonstrar o quanto este tem um consumo ecologicamente consciente? A preocupação ambiental que o consumidor tem afeta a sua consciência ambiental? O comportamento altruísta pode afetar o consumo ecologicamente consciente?

Nesse sentido, em 1999, Straughan e Roberts (1999) tentaram responder a essas perguntas validando uma escala denominada Ecologically Conscious Consumer Behavior (ECCB). Em razão da complexidade do tema e da evolução das relações consumidor-sustentabilidade-empresa, essas questões continuam sendo objeto de discussão e debate (ZIMMER; STAFFORD; STAFFORD, 1994; ROBERTS, 1996; STRAUGHAN; ROBERTS, 1999). Procurando reinterpretar os resultados dessa escala, no presente trabalho teve-se como objetivo analisar as interferências dos fatores presentes na escala $E C C B$ no consumo consciente de cosméticos. O uso da escala $E C C B$ justifica-se pelo crescimento das publicações na área de comportamento consumidor. Já a escolha do setor de cosméticos como objeto de estudo ocorreu pelos investimentos crescentes desse segmento em estratégias ecologicamente corretas.

Para resgatar a discussão em relação a essa escala, o presente artigo estudará o tema mediante uma abordagem pouco utilizada nos estudos da administração: a modelagem de segunda ordem. Essa abordagem se origina nos conjuntos de técnicas de validação empírica da modelagem de equações estruturais (MEE) e permite ao pesquisador testar 
estudos teóricos na prática, proporcionando uma avaliação do ajuste do modelo e dos parâmetros individuais, por meio da comparação entre modelos alternativos, para sugerir que uma especificação de ordem mais elevada seja mais adequada à relação teoria/prática estudada (GERBING; HAMILTON; FREEMAN, 1994; KOUFTEROS; BABBAR; KAIGHOBADI, 2009; LILJEBLOM; VAIHEKOSKI, 2009).

\section{O CONTEXTO DA ESCALA ECOLOGICALLY CONSCIOUS CONSUMER BEHAVIOR (ECCB)}

Nas últimas décadas, os produtos verdes e a consciência ambiental vêm alterando drasticamente a forma como as empresas investem. Nas décadas de 1960 e 1970, correntes ambientalistas defendiam a preservação dos recursos ambientais em detrimento do crescimento industrial e econômico. Logo após, na década de 1980, a corrente ambientalista passou a ser divulgada em grandes mídias, chegando a popularizar o conceito de marketing verde (STRAUGHAN; ROBERTS, 1999). Esse aumento da mídia beneficiou a preservação ambiental, pois os consumidores começaram a defender a necessidade de proteção do meio ambiente e passaram a perceber que o consumo exacerbado causava a redução dos recursos naturais (ROBERTS, 1996). Esse fato incentivou consumidores a mostrarem preferência a produtos ecologicamente corretos. O número de lançamentos de produtos verdes cresceu considervelmente a partir de 1985. Naquele ano, foram lançados 24 novos produtos verdes, que correspondiam a $0,5 \%$ dos novos produtos. Já no início da década de 1990, em 1991, foram lançados 810 novos produtos verdes no mercado, o que equivalia a 13,4\% de todas as inovações (OTTMAN, 1994).

Ainda na década de 1990, grandes empresas começaram a adotar práticas ecologicamente corretas. Esse segmento, que era exclusividade de pequenos nichos de empresas, começou a ser praticado por empresas de grande porte, como Walmart, McDonald's, Coca-Cola, Procter e Gamble, Móbil, entre outras. O início do século tem presenciado o aumento de campanhas e propagandas nos temas relacionados à proteção e conservação ambiental, aumentando, assim, as influências sobre atitudes e comportamentos do consumidor (PAÇO; RAPOSO, 2010). Com os crescentes níveis de consumo, movimentos de resistência ou anticonsumismo vêm se tornando mais populares, propondo modos de consumir mais conscientes do ponto de vista ecológico e social (HAANÄÄ, 2007).

Nesse contexto, diversos estudos têm abordado as características dos consumidores ecologicamente conscientes, tendo como foco de investigação 
indicadores comportamentais de compromisso ambiental ou escalas psicométricas de medição da consciência ambiental (ZIMMER; STAFFORD; STAFFORD, 1994; ROBERTS, 1996; STRAUGHAN; ROBERTS, 1999). Alguns desses estudos, como o de Roberts (1996), têm desenvolvido dimensões atitudinais ou psicográficas associadas a atitudes de comportamento do consumidor ecologicamente consciente.

A escala desenvolvida por Roberts (1996) foi denominada Ecologically Conscious Consumer Behavior (ECCB) e o seu uso como medida comportamental é essencial em razão da potencial diferença entre as atitudes ambientais e o real comportamento dos consumidores (STRAUGHAN; ROBERTS, 1999). Algumas variáveis manifestas foram selecionadas a partir de escalas existentes e outras foram desenvolvidas para refletir a natureza do consumo ecologicamente consciente. A variável dependente mede a extensão na qual a compra de bens e serviços tem um impacto positivo ou negativo em relação ao meio ambiente, considerada ECCB. De acordo com Roberts (1996), a orientação comportamental da escala ajuda a entender as atitudes, as quais, muitas vezes, não se traduzem em comportamento. Inicialmente, a escala de ECCB foi confeccionada com um total de 30 variáveis manifestas, sendo medidas por meio de uma escala Likert. Essas variáveis manifestas foram analisadas, reduzidas e segmentadas em três constructos: percepção da eficácia pelo consumidor, liberalismo e preocupação ambiental. Neste estudo, por sugestão de Straughan e Roberts (1999), foi incluído um quarto constructo, denominado comportamento altruísta.

O primeiro constructo, a percepção da eficácia pelos consumidores, é correspondente à capacidade do consumidor em crer que seu esforço vai fazer a diferença em termos da escassez dos recursos ambientais (PAÇO; RAPOSO, 2010). Nesse constructo, os indivíduos apresentam uma maior tendência em mostrar atitudes e comportamentos socialmente responsáveis. Nele, os consumidores conseguem entender o impacto de suas compras nos recursos naturais e na sociedade (BERGER; CORBIN, 1992; ROBERTS, 1996; STRAUGHAN; ROBERTS, 1999; PAÇO; RAPOSO, 2010). Por essa razão, é proposta a hipótese 1, descrita a seguir:

H1: A percepção da eficácia pelo consumidor afetará positivamente o consumo ecologicamente consciente de cosméticos.

Nas décadas de 1960 e 1970, observou-se a emergência de amplo apoio público para a preocupação ambiental, levando várias pessoas a se filiarem a partidos políticos que defendessem as causas ambientais. Essa corrente foi definida como a dos liberais. Desse modo, o segundo constructo, denominado liberalismo, 
apoia-se na corrente liberal, a qual se opõe às grandes empresas representantes do desenvolvimento industrial, incentiva reformas dos governos para auxiliar práticas ecológicas e procura ações inovadoras para o meio ambiente (ROBERTS, 1996). Estudos desenvolvidos nas últimas décadas apontam que as medidas de liberalismo têm evidenciado relações positivas com a preocupação ambiental (MITCHELL, 1983; SAMDAHL; ROBERTSON, 1989; ROBERTS, 1996; STRAUGHAN; ROBERTS, 1999). Assim, pode-se esperar que:

H2: O grau de liberalismo do consumidor afetará positivamente o consumo ecologicamente consciente de cosméticos.

O terceiro constructo, a preocupação ambiental, tem sido, muitas vezes, utilizado para rotular a responsabilidade social. Desse modo, cada vez mais consumidores estão exigindo que as empresas pratiquem atividades que demonstrem a preocupação com o meio ambiente. Nesse contexto, a preocupação ambiental pode ser um preditivo significativo da ECCB (ROBERTS, 1996; STRAUGHAN; ROBERTS, 1999; PAÇO; RAPOSO, 2010). Desse modo, espera-se que:

H3: A preocupação ambiental afetará positivamente o consumo ecologicamente consciente de cosméticos.

A importância relativa do altruísmo na previsão da $E C C B$ sugere que as empresas devem não somente ser explícitas na relação entre as suas estratégias ambientais e resultados benéficos, mas também devem mostrar como outras pessoas estão em melhor situação com os resultados da empresa. Esse achado corrobora os estudos de Stern, Dietz e Kalof (1993). No estudo de Roberts (1996), nenhuma ligação foi feita entre altruísmo e ECCB. No entanto, o autor sugere mensurar o altruísmo. Desse modo, o quarto constructo utilizado nesta pesquisa é o comportamento altruísta. Os resultados de Straughan e Roberts (1999) demonstram que a eficácia percebida do consumidor influencia diretamente o comportamento ecologicamente consciente. Além disso, a inclusão de altruísmo ao perfil parece aumentar significativamente os resultados encontrados. Desse modo, espera-se que:

H4: O comportamento altruísta afetará positivamente o consumo ecologicamente consciente de cosméticos. 


\section{1 ESTRUTURA HIERÁRQUICA DA ECCB COMO ABSTRAÇÃO DE SEGUNDA ORDEM}

Estudos que empregam técnicas avançadas de MEE, como modelagem de ordem superior, são escassas no meio acadêmico (KOUFTEROS; BABBAR; KAIGHOBANI, 2009). Na verdade, existem trabalhos acadêmicos que exemplificam a modelagem de estrutura de ordem superior, no entanto, existem poucas tentativas de apoiar conceitualmente esse processo e seu nível de abstração (KOUFTEROS; BABBAR; KAIGHOBANI, 2009).

Paradoxalmente, existem diversos constructos que podem ser facilmente conceituados em ordens superiores de abstração (KOUFTEROS; BABBAR; KAIGHOBANI, 2009). Em tais casos, uma abordagem de modelagem de ordem superior será a técnica mais adequada para representar tais estruturas. Considere-se, por exemplo, que o consumo ecologicamente consciente (como uma abstração de segunda ordem) pode ser conceituado como um composto de quatro variáveis latentes de segunda ordem, como eficácia, comportamento altruísta, preocupação ambiental e liberalismo.

Os consumidores de cosméticos podem avaliar esses constructos de primeira ordem por meio de variáveis manifestas, formando, assim, a opinião em relação ao constructo de segunda ordem, ou seja, o consumo ecologicamente correto. Dessa forma, as avaliações das variáveis latentes de primeira ordem agregam valor para entender melhor as percepções de um constructo de ordem superior geral. Nesse caso, um modelo de ordem mais elevada pode ser postulado e pode relacionar as variáveis manifestas às suas respectivas variáveis latentes de primeira ordem, que podem, então, ser relacionadas à sua variável latente de segunda ordem. Variáveis latentes de primeira ordem podem ser especificadas como dimensões de variáveis latentes de segunda ordem, se houver uma razão conceitual e teórica para tal relação. No caso deste artigo, essa razão conceitual e teórica é originada no artigo de Roberts (1996), publicado no Journal of Consumer Marketing, no qual é apresentada a escala denominada Ecologically Conscious Consumer Behavior (ECCB). Por meio dessa escala, o presente artigo apresenta um modelo de abstração de segunda ordem (Esquema 1). A adaptação da escala ECCB para um modelo de abstração de segunda ordem tem como intenção avaliar a sua relação com o consumo ambientalmente correto de cosméticos.

A vantagem de se ter construções de segunda ordem agrupando constructos de primeira ordem é que a explicação do modelo se torna mais completa (GERBING; HAMILTON; FREEMAN, 1994). Além de a modelagem de segunda ordem ser uma 
representação mais fidedigna das construções hierárquicas, ela pode servir para resolver problemas metodológicos que aparecem em estudos empíricos. Por exemplo, apesar de serem constructos diferentes (comportamento altruísta, eficácia, preocupação ambiental e liberalismo), eles podem ter variáveis observáveis altamente correlacionadas. No entanto, se todas as variáveis latentes estão no primeiro nível de abstração e são inseridas simultaneamente em um modelo que é postulado como antecedente da $E C C B$, um pesquisador vai logo descobrir que talvez apenas uma ou nenhuma dessas variáveis é estatisticamente relacionada à variável dependente; ou, ainda, observar que os coeficientes podem assumir o sinal oposto do que seria esperado. Isso pode ser sinal da multicolinearidade presente no modelo. Em uma estrutura individual, cada uma dessas variáveis observáveis pode ter uma relação estatisticamente significativa com uma variável endógena como a $E C C B$, mas, quando testado como um grupo de constructos de primeira ordem, tais efeitos não podem ser manifestados.

Construções altamente correlacionadas não produzem uma estrutura de fatores fidedigna. Embora a análise fatorial busque agrupar as variáveis observáveis, há uma grande probabilidade de que menos fatores significativos sejam extraídos, e os itens manifestos, assim, podem apresentar cargas não reais. Isso é preocupante, pois, dessa forma, em construções de primeira ordem, será difícil descrever de forma significativa os testes de hipóteses. Já as construções de segunda ordem mantêm a natureza idiossincrática de cada constructo de primeira ordem e tratam tais construções como partes do constructo de ordem superior.

Além das contribuições citadas, o emprego de ordem superior de modelagem também pode resolver problemas como o "inchaço" dos fatores, cunhado por Catell (1973) como bloated specifics factors. Esse "inchaço" acontece porque as variáveis latentes de primeira ordem são caracterizadas exclusivamente por uma extensão de conteúdo restrita às variáveis manifestas. Gerbing, Hamilton e Freeman (1994) explicam que, como os investigadores tentam satisfazer indicadores unidimensionais (especialmente quando testados por meio de metodologia de modelagem de equações estruturais sofisticadas), muitas vezes, eles inventam variáveis manifestas, as quais são reformulações mais ou menos semelhantes das outras variáveis manifestas. Enquanto essas variáveis manifestas podem satisfazer rigorosos critérios estatísticos e apresentar excelentes propriedades psicométricas e de domínio de conteúdo, tais variáveis são limitadas pelo poder explicativo e utilidade teórica (GERBING; HAMILTON; FREEMAN, 1994). Nesse contexto, os fatores de primeira ordem podem ser definidos por um conjunto unidimensional de itens e, assim, podem ser tratados como os indicadores de segunda ordem. O Esquema 1 representa, graficamente, o discutido: 
Esquema 1 - Estrutura hierárquica da $E C C B$

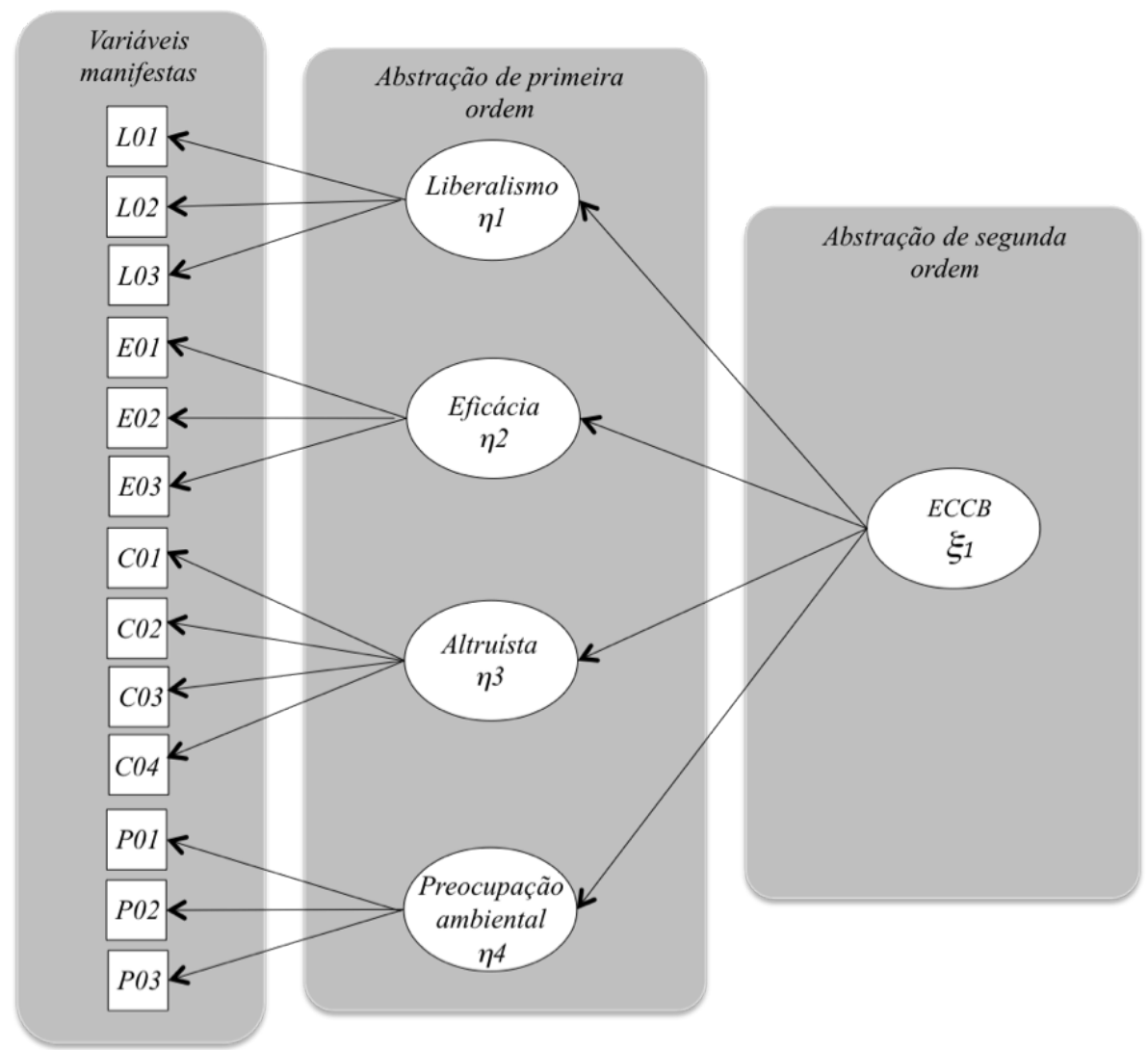

Fonte: os autores.

\section{DESIGN METODOLÓGICO, DESENVOLVIMENTO DO INSTRUMENTO E CARACTERÍSTICAS DA AMOSTRA}

Utilizou-se uma pesquisa descritiva para analisar as relações funcionais da $E C C B$ por meio da modelagem de segunda ordem. A pesquisa descritiva permite um estudo profundo e exaustivo de objetos, de maneira a permitir conhecimento amplo e detalhado destes (YIN, 2001). Ainda no escopo de delineamento, esta pesquisa se apresenta na abordagem quantitativa, sendo executada mediante uma pesquisa de campo.

Os dados primários foram coletados por intermédio de questionários estruturados adaptados em modelagem, de acordo com os estudos de Roberts (1996) e de Straughan e Roberts (1999), que analisam a escala ECCB. O questionário foi dividido em seis blocos 
de variáveis. O primeiro bloco continha perguntas envolvendo dados socioeconômicos, como: sexo, idade, renda, instrução, cidade onde residia e características gerais de consumo de cosmético (tipo de produtos mais consumidos, principais, dispêndio mensal com produtos, entre outros). Os blocos restantes foram desenvolvidos em uma escala de 1 (discordo plenamente) a 5 (concordo plenamente). Os blocos de número dois, três e quatro diziam respeito ao trabalho de Roberts (1996). O bloco dois avaliava o constructo liberalismo e continha um total de três variáveis manifestas. O bloco três avaliava o constructo eficácia e continha um total de três variáveis manifestas. O bloco quatro avaliava o constructo preocupação ambiental e continha um total de três variáveis manifestas. O bloco de número cinco avaliava o constructo altruísmo, sugerido por Straughan e Roberts (1999) para complementar os estudos de Roberts (1996). Esse constructo teve um total de quatro variáveis manifestas. O último bloco avaliava a variável endógena consumo ambiental de cosméticos, com um total de três variáveis manifestas. Para a elaboração desse último bloco, foram utilizados dados secundários extraídos da internet e de pesquisa bibliográfica.

Os questionários foram respondidos por amostragem não probabilística por conveniência. Participaram do estudo 554 consumidores de cosméticos da região metropolitana de Porto Alegre. O período de coleta dos dados foi de seis meses, com início no mês de junho e término em dezembro de 2011. Antes, porém, foi aplicado um pré-teste com cinco consumidores que comprovaram a eficiência (do instrumento), a fim de que eles projetassem suas próprias concepções e valores a respeito do tema proposto. A amostra coletada revela-se representativa, pois, segundo Bentler e Chou (1987), pesquisas empíricas que utilizam modelagem devem contar com um número entre 5 e 10 casos por parâmetro estimado. No total, foram avaliadas 16 questões por 554 respondentes, o que gera um valor de 34,625 casos por parâmetro estimado.

Para interpretar os objetos deste estudo, foi utilizado o método de Modelagem de Equações Estruturais, como aplicabilidade dos conceitos de modelagem de segunda ordem, segundo estudos de Koufteros, Babbar e Kaighobani (2009) e Jarvis, Mackenzie e Podsakoff (2003). A técnica de Modelagem de Equações Estruturais, do inglês Structural Equation Modeling (SEM), é construída por meio de uma família de procedimentos estatísticos relacionados (KLINE, 2010; HAIR et al., 2009). A escolha da MEE de segunda ordem deve-se ao fato de existir uma escassez desse tipo de análise em estudos brasileiros. Esse método auxilia na análise de dados não experimentais (correlacionais) e experimentais, possibilitando uma flexibilidade nas relações múltiplas analisadas. A análise dos modelos ocorreu por meio do software AMOS 18.0. 


\section{ANÁLISE DE RESULTADOS}

Como foi descrito na revisão da literatura, o consumo consciente pode ser influenciado por diversos fatores. Nesse sentido, o estudo em questão analisou o consumo consciente de cosméticos com base nos fatores que influenciam na escala ECCB. Para melhor explicitar os resultados obtidos, esta seção foi dividida em cinco partes: análise preliminar das estatísticas descritivas; validação individual dos constructos; abordagem hierárquica e a construção de Modelos de Medição Alternativos; análise dos modelos alternativos; e, modelo estrutural final e a influência no consumo consciente de cosméticos.

\section{1 ANÁLISE PRELIMINAR DAS ESTATÍSTICAS DESCRITIVAS}

Por meio da análise estatística descritiva dos 554 questionários respondidos, identificou-se que $72,2 \%$ dos respondentes são do sexo feminino e que grande parte tem menos de 30 anos (72,6\%); 58,2\% têm o ensino superior completo e outros $32,2 \%$ estão em curso; $59,2 \%$ são solteiros e $72,4 \%$ ganham menos de R $\$ 3.000,00$. Em termos relativos, esses dados evidenciam o público-alvo da pesquisa.

Em relação à marca de cosméticos, 69,7\% consomem a marca Natura e $55 \%$ consomem O Boticário. Apenas $4 \%$ mencionaram consumir outras marcas de cosméticos. No que se refere aos tipos de produtos, $44,6 \%$ têm o hábito de consumir xampu e $63 \%$, hidratantes. O gasto médio mensal da amostra com cosméticos ficou em torno de $\mathrm{R} \$ 50,00$ a $\mathrm{R} \$ 100,00$.

Por ser considerada uma forma de consumo heterogêneo por parte dos profissionais de varejo, poderia ser encontrado um viés de erro nas respostas, principalmente ao se relacionar sexo a consumo. No entanto, não se pode considerar que existe uma diferença significativa da relação entre sexo e consumo de cosméticos, pois por meio do teste Qui-quadrado foi comprovado que se deve aceitar a hipótese nula (Ho), em razão de esse índice ser maior que 0,05. 


\section{2 VALIDAÇÃO INDIVIDUAL DOS CONSTRUCTOS}

Partindo do modelo apresentado na base teórica, os cinco constructos seriam formados por três ou quatro variáveis manifestas cada um, dependendo de sua construção inicial. No caso da relação entre as variáveis latentes e os constructos latentes, os coeficientes estimados oferecem informações sobre a extensão na qual determinada variável manifesta é capaz de medir uma variável latente (SCHUMACKER; LOMAX, 1996; ANDERSON; GERBING, 1988). Observa-se que os coeficientes estimados apresentaram valores positivos e significativos, evidenciando que os indicadores estão positivamente relacionados aos constructos. Os índices de ajuste do modelo foram adequados também na análise inicial, porém, o constructo eficácia não teve coeficientes significativos com as suas respectivas variáveis manifestas.

Em uma primeira análise, fica evidenciado que o teste de Qui-quadrado é significativo para todos os constructos, indicando que há uma diferença significativa entre a matriz observada e a matriz estimada. As medidas absolutas de ajuste, que determinam o grau em que o modelo prediz a matriz de covariância observada, apresentam valores dentro dos limites aceitáveis. A confiabilidade ficou acima de 0,5 na maior parte dos casos, com o nível de probabilidade inferior a 0,05, indicando que a consistência interna entre os indicadores é satisfatória. Somente no caso do constructo eficácia, os coeficientes e a significância não alcançaram os valores esperados. Diante desses resultados iniciais, decidiu-se modificar o modelo. Seguindo a literatura sobre equações estruturais, optou-se, então, por retirar o constructo eficácia do modelo teórico inicial, promovendo alterações para ajuste deste. Esse procedimento está de acordo com a proposição de que devem ser retiradas as variáveis manifestas cujos coeficientes estejam entre os menores ou não sejam significativos, ou seja, que obtiverem valores menores que 0,5 (KLINE, 2010).

Após ajuste no modelo, os índices de ajuste absolutos e comparativos apresentaram resultados melhores do que os valores mínimos sugeridos. Em relação à fidedignidade e confiabilidade do constructo, a variância extraída e a confiabilidade ficaram acima do limite sugerido em quase todas as análises, visto que, na análise do Alpha de Cronbach, obtiveram índice favorável acima de 0,7. Em um primeiro momento, verificou-se a estrutura discriminante e convergente dos constructos. Os resultados das cargas fatoriais dos itens sobre cada variável latente evidenciaram loadings significativos a 0,01. A Tabela 1 apresenta os resultados. 
Tabela 1 - Índices de confiabilidade dos constructos

\begin{tabular}{|c|c|c|c|c|c|}
\hline Constructos & $\begin{array}{l}\text { Liberalis- } \\
\text { mo }\end{array}$ & Eficácia & $\begin{array}{l}\text { Altru- } \\
\text { ísta }\end{array}$ & $\begin{array}{l}\text { Preocu- } \\
\text { pação } \\
\text { ambiental }\end{array}$ & $\begin{array}{l}\text { Consumo } \\
\text { consciente de } \\
\text { cosméticos }\end{array}$ \\
\hline Liberalismo & $\begin{array}{l}, 831^{\star} \\
, 621^{\star \star}\end{array}$ & & & & \\
\hline Eficácia & $\mathrm{Nd}$ & nd & & & \\
\hline Altruísta & ,012 & nd & $\begin{array}{l}, 884^{\star} \\
, 718^{\star \star}\end{array}$ & & \\
\hline $\begin{array}{l}\text { Preocupação } \\
\text { ambiental }\end{array}$ & ,169 & nd & ,143 & $\begin{array}{l}, 932^{\star} \\
, 821^{\star \star}\end{array}$ & \\
\hline $\begin{array}{l}\text { Consumo } \\
\text { consciente de } \\
\text { cosméticos }\end{array}$ & ,179 & nd & ,638 & ,142 & $\begin{array}{l}, 946^{\star} \\
, 815^{\star \star}\end{array}$ \\
\hline
\end{tabular}

Fonte: os autores.

Nota: ${ }^{\star}$ Confiabilidade Composta; ${ }^{\star \star}$ Variância Extraída

Os indicadores de Confiabilidade simples (Alfa de Crombach) e de Variância Média Extraída (AVE), este proposto por Fornell e Larcker (1982), foram considerados significativos. Observando os resultados da AVE de cada constructo, na Tabela 1 da matriz de correlação, nota-se que esses resultados são sempre maiores do que as correlações entre os constructos, sendo todas elas significativas, com índices não muito altos (acima de 0,90), o que caracterizaria multicolinearidade (HAIR et al., 2009; SCHUMACKER; LOMAX, 1996). É importante analisar esse critério, pois, segundo Fornell e Larcker (1982), esses resultados viabilizam a verificação da validade convergente para cada constructo, pelo fato de a AVE ser maior que a variância compartilhada (VC).

\section{3 ABORDAGEM HIERÁRQUICA E CONSTRUÇÃO DE MODELOS DE MEDIÇÃO ALTERNATIVOS}

O primeiro passo para analisar a abordagem hierárquica é verificar as possíveis alternativas de modelos de mensuração sugeridos para descrever os dados. Segundo Koufteros, Babbar e Kaighobani (2009), esse passo se torna necessário, pois podem existir várias configurações de medição de modelos que representam diferentemente determinados conjuntos de dados (MARSH; HOCEVAR, 1985; RINDSKOPF; ROSE, 1988; GERBING; HAMILTON; FREEMAN, 1994). Desse modo, faz-se necessário um estudo da natureza hierárquica dos dados coletados. 
A abordagem hierárquica é um processo sistemático de avaliação de modelos alternativos que têm o potencial para descrever relações entre variáveis manifestas e latentes. Esse processo inclui a construção de quatro modelos (KOUFTEROS; BABBAR; KAIGHOBANI, 2009). O avanço conceitual dos quatro modelos tem como objetivo fornecer um delineamento explícito dos domínios de conteúdo que operacionalizam as construções de interesse (GERGING; HAMILTON; FREEMAN, 1994). O Esquema 2 apresenta os dois primeiros modelos testados.

Esquema 2 - Modelos 01 e 02

Modelo 01: Um fator de primeira ordem

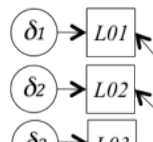

(d3) $>L 03$

(64) $>E 01$

(D5) $>E 02 k$

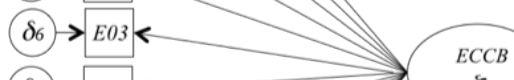

(d7) $>\mathrm{CO1}<$

(88) $>\mathrm{CO}<$

(d9) $\rightarrow \mathrm{CO}^{2}$

(ס10) $>\mathrm{CO4}$

(S11) $>P_{01}$

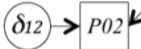

(D13) $>\mathrm{P03}$

Fonte: os autores.

$\xi 1$
Modelo 02: Quatro fatores ortogonais de primeira ordem (não correlacionados)
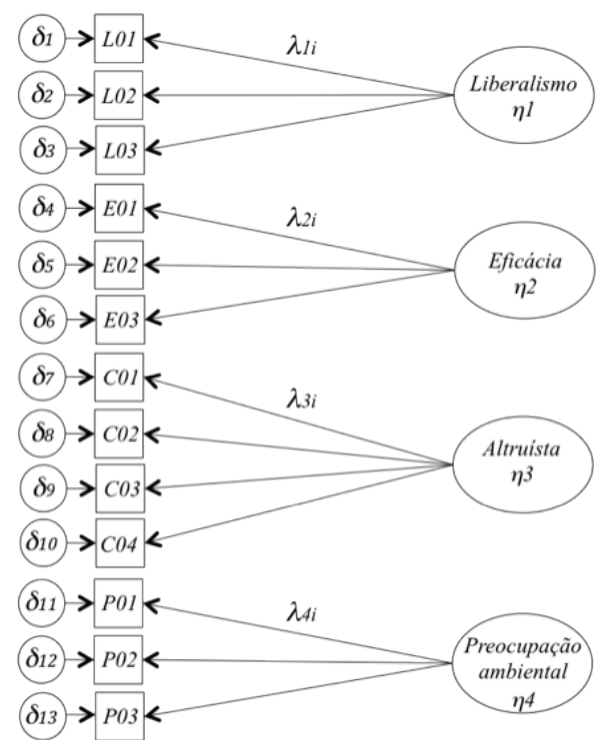

O primeiro modelo refere-se à construção de uma variável latente de primeira ordem, por meio das relações diretas com as variáveis manifestas encontradas no estudo. Nesse caso, tal modelo é determinado como de primeira ordem e é originado pelas 13 variáveis manifestas do estudo e a variável latente ECCB. O segundo modelo refere-se à construção que envolve os quatro constructos de primeira ordem correlacionados, sendo denominado ortogonal. Nesse modelo, as quatro variáveis latentes (liberalismo, eficácia, altruísmo e preocupação ambiental) não são correlacionadas e são formadas diretamente por suas respectivas variáveis manifestas, segundo a escala ECCB. 
O terceiro modelo é semelhante ao segundo, tendo como diferença central a correlação entre os quatros constructos latentes especificados da escala $E C C B$ (liberalismo, eficácia, altruísmo e preocupação ambiental). Com base em suas características, esse modelo foi denominado quatro fatores de primeira ordem correlacionados. Por fim, o quarto modelo inclui um fator de segunda ordem $(E C C B)$ e quatro de primeira ordem (liberalismo, eficácia, altruísmo e preocupação ambiental), com as respectivas variáveis latentes. Esse último tipo de modelo de segunda ordem, que mostra os indicadores de primeira e de segunda ordem, sendo reflexivo, é frequentemente encontrado na literatura (JARVIS; MACKENZIE; PODSAKOFF, 2003) e denominado modelo de desagregação total de segunda ordem, do inglês total disaggregation second-order factor model (BAGOZZI; HEARTHERTON, 1994). Nesse caso, o fator de segunda ordem é uma hipótese para explicar toda a covariância entre os fatores de primeira ordem (JÖRESKOG, 1970). O terceiro e o quarto modelos são descritos no Esquema 3.

Após selecionar os modelos de medição, o próximo passo é testar as hipóteses que fundamentam cada um e, com isso, especificar os quatro modelos. Os modelos estruturais são avaliados por meio dos t-values dos coeficientes (estes são utilizados para testar as hipóteses de pesquisa). As magnitudes dos coeficientes estruturais e a sua significância estatística são úteis na avaliação de possíveis relações entre as variáveis. Além disso, uma análise plausível contempla também uma avaliação de $r$-squared e dos vários índices de ajustamento que podem ser comparados, como: qui-quadrado/graus de liberdade, normed fit index (NFI), comparative fit index (CFI), non-normed fit index (NNFI) e standardized root mean square residual (RMSEA) (KOUFTEROS; BABBAR; KAIGHOBANI, 2009). O Esquema 3 apresenta os modelos 03 e 04, anteriormente citados. 
Esquema 3 - Modelos 03 e 04 Modelo 03: Quatro fatores de primeira ordem correlacionados

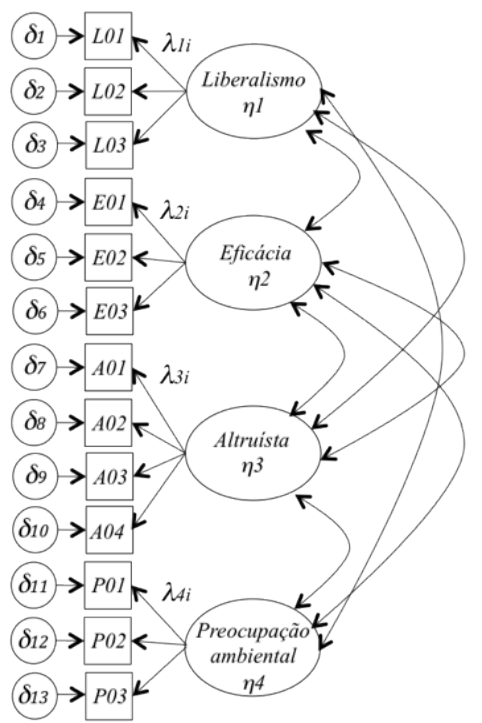

Modelo 04: Quatro fatores de primeira ordem com um fator de segunda ordem

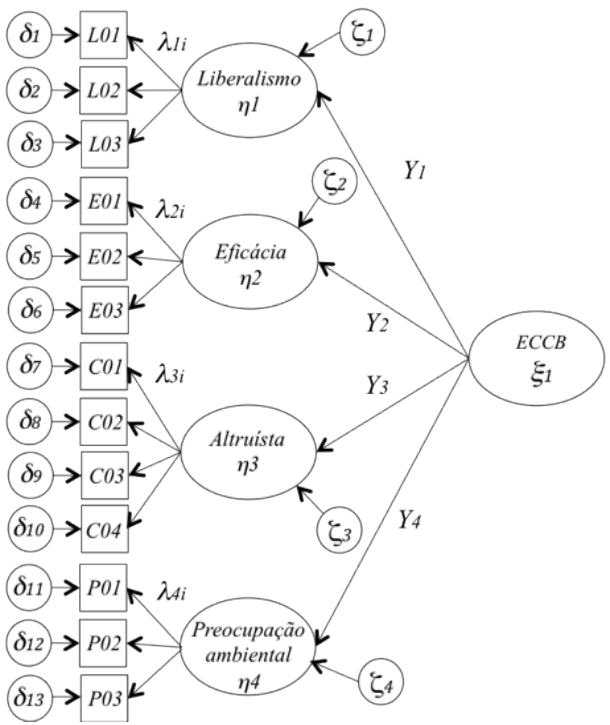

Fonte: os autores.

Nota: O modelo 03 possui duas correlações entre altruísta e eficácia.

Cabe ressaltar que a escolha final do modelo de mensuração não pode ser pautada na mera comparação entre os índices de ajustamento. Esses modelos são sustentados teoricamente. Dessa forma, os índices são considerados apenas cortes iniciais. Isso significa que os modelos que apresentam índices baixos de ajustamento não devem avançar para as próximas fases de análise. Por outro lado, um modelo de medição que gera melhores índices de ajustamento também não pode, automaticamente, assegurar sua posição como o modelo principal. Questões como validade discriminante, grau de multicolinearidade e identificação do modelo devem ser consideradas também (RINDSKOPF; ROSE, 1988; KOUFTEROS; BABBAR; KAIGHOBANI, 2009).

\section{4 ANÁLISE DOS MODELOS ALTERNATIVOS}

O primeiro modelo especifica que todos os 10 itens são reflexivos de uma variável latente, no caso, $E C C B$. A relação $\chi 2 /$ df resultou maior que cinco e os índices de ajustamento tiveram valores baixos (NFI=,596; IFI=,607; CFI=,603; RMSEA =,189), indicando ajustes não muito bons. Essa adequação do modelo 
demonstrou resultados não favoráveis à combinação de variáveis manifestas, a partir de diferentes domínios de conteúdo, a uma variável latente de primeira ordem. Embora possam ser correlacionados, os itens de cada domínio podem ser distintos e não refletir uma especificação ideal, como é representado na Tabela 2. Desse modo, constata-se que, nesta pesquisa, a combinação de variáveis latentes distintas em uma construção de primeira ordem única é conceitualmente e metodologicamente inapropriada.

Tabela 2 - Índices dos modelos alternativos

\begin{tabular}{lllll}
\hline $\begin{array}{l}\text { Índices } \\
(\mathbf{n}=\end{array}$ & $\begin{array}{l}\text { Modelo 01: } \\
\text { Um fator } \\
\text { de primeira } \\
\text { ordem }\end{array}$ & $\begin{array}{l}\text { Modelo 02: } \\
\text { Quatro fatores } \\
\text { ortogonais de } \\
\text { primeira ordem } \\
\text { (não correla- } \\
\text { cionados) }\end{array}$ & $\begin{array}{l}\text { Modelo 03: Qua- } \\
\text { tro fatores de } \\
\text { primeira ordem } \\
\text { correlacionados }\end{array}$ & $\begin{array}{l}\text { Modelo } \\
\text { 04: Quatro } \\
\text { fatores de pri- } \\
\text { meira ordem } \\
\text { com um fator } \\
\text { de segunda } \\
\text { ordem }\end{array}$ \\
\hline$\chi 2(\mathrm{df})$ & $998,248(36)$ & $61,33(1)$ & $79,965(32)$ & $97,156(33)$ \\
$\chi 2 / \mathrm{DF}$ & 27,72 & 61,33 & 2,49 & 2,94 \\
NFI &, 451 &, 889 &, 956 &, 947 \\
IFI &, 460 &, 891 &, 924 &, 964 \\
CFI &, 454 &, 890 &, 973 &, 964 \\
RMSEA &, 219 &, 329 &, 052 &, 059 \\
\hline
\end{tabular}

Fonte: os autores.

O segundo modelo postula três variáveis latentes não correlacionadas que estão relacionadas às suas respectivas variáveis manifestas. Considerando as altas correlações entre as variáveis manifestas e latentes, como notado na análise fatorial inicial, pode-se esperar uma especificação ortogonal com relações entre as variáveis latentes com ajustes do modelo adequado. Na verdade, tal fato ocorre em razão de as correlações entre variáveis manifestas e latentes terem sido altas. No entanto, os índices não demonstraram valores recomendados pela literatura de modelagem de equações estruturais ( $\mathrm{NFI}=, 889$; $\mathrm{IFI}=, 891$; $\mathrm{CFI}=, 890 ; \mathrm{RMSEA}=, 329)$. Desse modo, apesar das altas correlações, os índices de ajustamento inviabilizam os fatores ortogonais de primeira ordem não correlacionados.

O modelo 03 tem as mesmas características do modelo 02, com exceção do fato de que as variáveis latentes estão livres para se correlacionar. A adequação do modelo foi bastante aceitável, pois todos os índices de ajustamento preencheram os critérios recomendados pela literatura (NFI=,956; IFI=,924; CFI=,973; RMSEA =,052). O modelo não correlacionado apresenta uma evolução na questão da validade discriminante, que foi um dos principais problemas do modelo correlacionado. Porém, cabe ressaltar que, quando os fatores de primeira ordem são 
altamente correlacionados, pode ocorrer a multicolinearidade, que é uma questão crítica na literatura de modelagem de equações estruturais. Todavia, quando os fatores de primeira ordem apresentam correlações moderadas, a especificação do modelo com quatro fatores de primeira ordem correlacionados seria aconselhável.

O modelo 04 apresenta um fator de segunda ordem (ECCB) que está relacionado a quatro fatores de primeira ordem, os quais, por sua vez, estão relacionados às respectivas variáveis manifestas. Dentro do contexto de ordem superior, a modelagem dos fatores de primeira ordem equipara-os a variáveis dependentes. Isso implica que as suas variâncias e covariâncias não sejam estimáveis dentro do modelo. Pelo contrário, presume-se que tal variação seja explicada pelo fator de ordem superior (BYRNE, 1998).

De acordo com a literatura (MARSH; HOCEVAR, 1985; ARNAU; THOMPSON, 2000), uma modelagem que inclui uma estrutura do modelo de segunda ordem nunca pode produzir um ajuste melhor (isto é, uma melhor adaptação em termos de índices de ajustamento) que um modelo que especifica somente a primeira ordem dos fatores correlacionados (como o modelo 03). No entanto, um fator de segunda ordem pode ser uma alternativa atrativa ao modelo de primeira ordem correlacionado. Observa-se que o modelo 04 é bem ajustado e não é muito divergente do desempenho do modelo 03 . Considerando-se a deficiência dos outros modelos (por exemplo, a validade discriminante e as questões de multicolinearidade dos modelos 02 e 03), fica evidenciada a necessidade de modelos de segunda ordem, justificados teoricamente e com o apoio da literatura de ECCB (ROBERTS, 1996). Desse modo, o modelo 04 aparenta ser a escolha mais prudente.

Examinando mais detalhadamente o modelo 04 e o conjunto de correlações constantes no modelo, os índices de ajuste, com os valores $t$, fornecem evidências de validade convergente. O NFI foi de ,947; IFI foi de ,964 e CFI foi de ,964; enquanto a relação qui-quadrado/grau de liberdade foi de 2,94. A RMSEA foi de ,059. Todos os itens têm relações estatisticamente significativas com os seus fatores. Todas as cargas fatoriais foram maiores que, 48 . Os valores $t$, associados aos fatores de item de cargas, excedem o valor crítico ao nível de significância 0,001. Observa-se que as cargas dos fatores de primeira ordem, para o fator de segunda ordem, estão todas acima de 0,78 e que os valores $t$ são estatisticamente significativos. Isso aponta para uma relação forte entre os fatores de primeira ordem e o fator de segunda ordem, atestando a validade convergente do modelo posto de segunda ordem. Desse modo, um modelo de segunda ordem é bastante eficaz na representação dos dados. 


\section{5 MODELO ESTRUTURAL FINAL E A INFLUÊNCIA NO CONSUMO CONSCIENTE DE COSMÉTICOS}

Uma vez selecionado o modelo 04 como o melhor de mensuração, procede-se com o teste do modelo estrutural. Nesse caso, postula-se um modelo estrutural, no qual o consumo consciente de cosméticos é especificado como a variável dependente, e o fator de segunda ordem (ou seja, ECCB) é a hipótese que irá afetá-lo. Na Tabela 3 são apresentadas a carga fatorial e a comunalidade das variáveis mensuradas na avaliação individual do modelo final.

Tabela 3 - Carga fatorial e comunalidades do modelo final (continua)

\begin{tabular}{|c|c|c|c|}
\hline Código & Descrição das variáveis & $\begin{array}{l}\text { Carga } \\
\text { fatorial }\end{array}$ & Comunalidade \\
\hline \multicolumn{4}{|c|}{ Liberalismo } \\
\hline L01 & $\begin{array}{l}\text { O lucro das grandes empresas deve ser } \\
\text { controlado pelo governo federal. }\end{array}$ & 876 &, 568 \\
\hline L02 & $\begin{array}{l}\text { Se o desemprego é alto, o governo deve } \\
\text { gastar para criar empregos. }\end{array}$ & ,673 &, 591 \\
\hline L03 & $\begin{array}{l}\text { Um governo seguro é necessário para ga- } \\
\text { rantir assistência médica adequada. }\end{array}$ & ,691 & ,489 \\
\hline \multicolumn{4}{|l|}{ Eficácia } \\
\hline E01 & $\begin{array}{l}\text { É inútil para os consumidores individuais } \\
\text { fazer qualquer coisa sobre a poluição. }\end{array}$ & ,782 & ,642 \\
\hline E02 & $\begin{array}{l}\text { Quando compro produtos, tento pensar } \\
\text { como o uso deles afetará o meio ambiente. }\end{array}$ & 661 &, 574 \\
\hline E03 & $\begin{array}{l}\text { Se uma pessoa não pode fazer nada sobre a } \\
\text { poluição, não faz diferença o que eu faço. }\end{array}$ & ,649 & ,682 \\
\hline \multicolumn{4}{|l|}{ Altruísta } \\
\hline $\mathrm{C} 01$ & $\begin{array}{l}\text { Tento comprar o máximo de produtos que } \\
\text { podem ser reciclados. }\end{array}$ &, 557 & ,497 \\
\hline $\mathrm{C} 02$ & $\begin{array}{l}\text { Compro produtos com o menor preço, } \\
\text { independentemente do seu impacto na } \\
\text { sociedade. }\end{array}$ & 691 &, 557 \\
\hline $\mathrm{C} 03$ & $\begin{array}{l}\text { Não compro produtos de uso doméstico } \\
\text { que prejudicam o meio ambiente. }\end{array}$ & ,678 & 693 \\
\hline $\mathrm{C} 04$ & $\begin{array}{l}\text { Para manter uma economia sustentável, o } \\
\text { crescimento industrial deve ser controlado. }\end{array}$ &, 599 & ,683 \\
\hline \multicolumn{4}{|c|}{ Preocupação ambiental } \\
\hline P01 & $\begin{array}{l}\text { Plantas e animais existem, principalmente, } \\
\text { para serem usados por seres humanos. }\end{array}$ & 695, & ,582 \\
\hline P02 & $\begin{array}{l}\text { Estamos nos aproximando do limite do nú- } \\
\text { mero de pessoas que a Terra pode suportar. }\end{array}$ & ,535 &, 571 \\
\hline
\end{tabular}


(conclusão)

\begin{tabular}{llll}
\hline Código & \multicolumn{1}{c}{ Descrição das variáveis } & $\begin{array}{c}\text { Carga } \\
\text { fatorial }\end{array}$ & Comunalidade \\
\hline P03 & $\begin{array}{l}\text { Para manter uma economia sustentável, o } \\
\text { crescimento industrial deve ser controlado. }\end{array}$ &, 672 &, 604 \\
\hline Consumo ambiental de cosméticos & Não compro cosméticos de empresas que &, 561 &, 515 \\
\hline CAC01 & $\begin{array}{l}\text { não protejam o meio ambiente } \\
\text { Compro cosméticos pelo preço e qualidade } \\
\text { percebida, sem considerar a causa ambien- }\end{array}$ &, 803 &, 617 \\
CAC02 & $\begin{array}{l}\text { tal. } \\
\text { CAC03 }\end{array}$ & $\begin{array}{l}\text { Prefiro cosméticos de empresas que respei- } \\
\text { tam o meio ambiente. }\end{array}$ &, 757 \\
\hline
\end{tabular}
Fonte: os autores.

Observa-se que a adequação do modelo atende às diretrizes geralmente aceitas pela literatura de modelagem de equações estruturais (MULAIK et al., 1989). O quiquadrado/grau de liberdade foi de 4,25 e o NFI foi de 0,87 . Ambos IFI $(0,88)$ e CFI $(0,88)$ não estão acima de 0,90 , porém, estão perto desse valor, podendo ser aceitos de acordo com os critérios do pesquisador. Resultado semelhante ao RMSEA, que não foi menor que 0,008 , tendo o valor de 0,074 . $\mathrm{O}$ t-valor, associado à relação entre $E C C B$ e consumo consciente de cosméticos, foi 7,248 e é bastante significativo. O modelo explicou $55,8 \%$ da variação do consumo consciente de cosméticos. A relação ECCB e consumo consciente de cosméticos teve um efeito de ,544. Coletivamente, as três variáveis tiveram um impacto significativo no consumo consciente de cosméticos. Entre os fatores de primeira ordem, o que obteve um efeito mais forte sobre a ECCB foi o liberalismo, com o valor de ,656. Logo após, o altruísmo teve um efeito moderado de ,579. Por fim, a preocupação ambiental gerou um efeito de ,446 sobre a ECCB. Contrariamente aos estudos de Roberts (1996), a relação liberalismo e ECCB apresentou coeficientes altos. No entanto, Roberts (1996) declara em seu estudo que, embora essa variável mostre relações menores, ainda é útil como uma variável de entendimento da ECCB. No caso do consumo consciente de cosméticos, esse constructo de primeira ordem se mostrou importante. Essa relação demonstra que as medidas de liberalismos estão presentes atualmente, pois os consumidores se mostraram contrários às grandes empresas e incentivam reformas dos governos para auxiliar práticas ecológicas para proteger o meio ambiente. De forma semelhante aos estudos de Mitchell (1983), Sandahl e Robertson (1989), Roberts (1996) e Straughan e Roberts (1999), o presente estudo aponta que as medidas de liberalismos têm evidenciado relações positivas com a ECCB. 
Tabela 4 - Coeficientes padronizados e significância do modelo integrado

\begin{tabular}{llllllll}
\hline \multicolumn{2}{c}{ Constructos } & & \multicolumn{1}{c}{$\begin{array}{c}\text { Coeficiente } \\
\text { não } \\
\text { padronizado }\end{array}$} & $\begin{array}{l}\text { Desvio } \\
\text { padrão }\end{array}$ & t-valor & \\
\hline Preocupação ambiental & $\leftarrow$ & Liberalismo &, 656 & 0,036 & 9,231 & * \\
Preocupação ambiental & $\leftarrow$ & Eficácia &, 579 & 0,011 & 13,674 & * \\
Preocupação ambiental & $\leftarrow$ & Altruísta &, 446 & 0,024 & 9,814 & * \\
$\begin{array}{l}\text { Consumo ambiental de } \\
\text { cosméticos }\end{array}$ & $\leftarrow$ & $\begin{array}{l}\text { Preocupação } \\
\text { ambiental }\end{array}$ &, 544 & 0,089 & 7,248 & * \\
\hline
\end{tabular}

Fonte: os autores.

Nota: ${ }^{\star}$ Valores acima de ,05.

No caso do efeito da preocupação ambiental na ECCB (embora significativo), este foi moderado, o que sugere que outros fatores desempenham um papel mais importante para explicar a probabilidade de um consumidor realizar tais comportamentos. Para Stisser (1994), ainda aparece que qualidade, preço, conveniência e valor são os critérios mais importantes de compra, deixando a preocupação ambiental em segundo plano.

A relação do altruísmo na $E C C B$ apresentou um coeficiente relativamente forte, demonstrando que as empresas devem não somente ser explícitas na relação entre as suas estratégias ambientais e resultados benéficos, mas, também, mostrar como outras pessoas estão em melhor situação em razão dos resultados da empresa, segundo menciona o estudo de Stern, Dietz e Kalof (1993). O Esquema 4 ilustra o modelo utilizado nesta pesquisa, o qual buscou preencher lacuna nos estudos de Roberts (1996) e Straughan e Roberts (1999), fazendo uma ligação entre altruísmo e ECCB. 
Esquema 4 - Modelo final

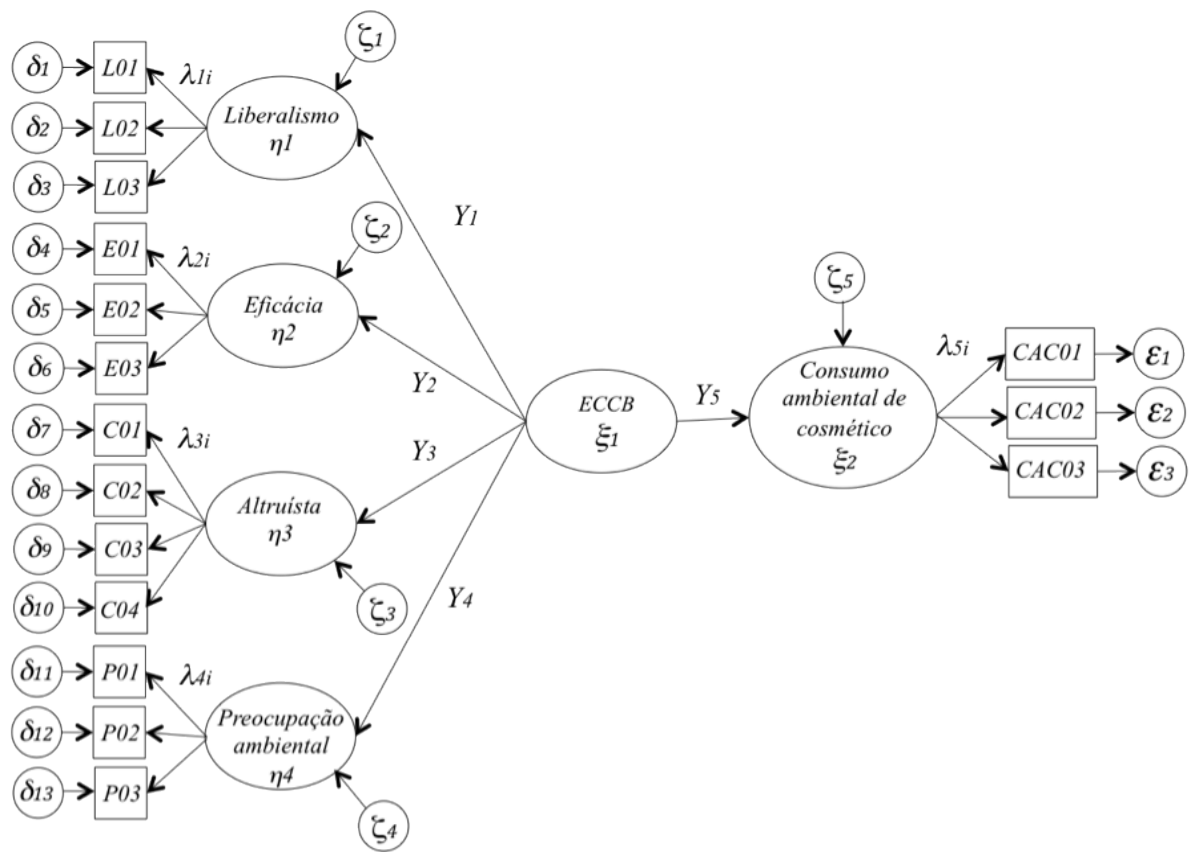

Fonte: os autores.

Ressalta-se que a interferência da percepção da eficiência não pode ser avaliada neste estudo. Contrariamente, a percepção da eficiência no consumo (ecologicamente consciente) foi identificada como a variável mais promissora para explicar a variação na ECCB do modelo de Roberts (1996). Desse modo, pesquisas futuras devem se concentrar sobre os possíveis antecedentes da percepção da eficiência no consumo e no desenvolvimento das estratégias mais eficazes para combater a percepção negativa da eficiência no consumo. Especificamente, os resultados do estudo de Roberts (1996) sugerem que um indivíduo deve ser convencido de que suas ações pró-ambientais serão eficazes na luta contra a deterioração ambiental. Isso tem implicações para uma variedade de atividades de marketing. Ele sugere que os esforços ambientais baseados em marketing devem ser explicitamente vinculados com resultados benéficos. 


\section{CONSIDERAÇÕES FINAIS}

As pesquisas que mensuram o consumo consciente ambiental se desenvolveram bastante nos últimos 20 anos, com diversos estudos empíricos realizados. Nesse contexto, apareceram diversas metodologias de análise de dados. O presente trabalho procurou ofertar mais uma técnica de análise nesse campo de estudo: a modelagem de segunda ordem.

Com essa técnica, os constructos que formam o consumo ambiental de cosméticos foram conceituados de forma mais significativa. Pode-se observar que a abordagem hierárquica para avaliar se uma conceituação de ordem superior é prudente, uma vez que outras estruturas poderiam também representar os dados de maneiras diferentes. Essa abordagem envolve uma avaliação rigorosa dos coeficientes e do modelo de mensuração (KISHTON; WIDAMAN, 1994). Assim, a variância dos coeficientes modelos de medidas deve ser avaliada antes do teste de variância do modelo estrutural. Desse modo, pode-se considerar uma especificação de segunda ordem como forma de agregação. A agregação é útil, pois pode representar as relações entre as variáveis mais parcimônicas. Por exemplo, variáveis manifestas podem ser representadas por uma única variável latente de primeira ordem. Da mesma forma, um fator de segunda ordem pode incorporar o significado de muitas variáveis latentes de primeira ordem. Assim, o número de variáveis que devem ser estimadas no contexto de um modelo estrutural pode ser reduzido significativamente (BAGOPZZI; HEATHERTIN, 1994; COFFMAN; MACCALLUM, 2005).

A motivação para o uso da modelagem de segunda ordem foi primeiramente conceitual, na qual o interesse científico central se coloca na análise geral da ECCB e de sua influência no consumo consciente de cosméticos. O fator de ordem superior foi a hipótese que explicou a relação entre os fatores de ordem inferior. Os resultados gerados nos modelos hierárquicos deste estudo podem ser comparados às investigações atitudinais da ECCB realizadas na década de 1990. Os resultados demonstraram que algumas relações se modificaram, como, por exemplo, a eficácia no consumo não pode ser verificada neste novo estudo. Além disso, foi incorporado o constructo de primeira ordem, altruísmo, que tinha sido sugerido em outros estudos de ECCB.

Novas análises são estimuladas, visando superar as limitações deste estudo. Entre as limitações, pode ser citado o fato de a amostra não ser probabilística e de o local de atuação se restringir à grande Porto Alegre. No que se refere a estudos futuros, investigações em outros estados e países permitiriam comparar os resultados em 
razão das especificidades locais, bem como a incorporação de outras variáveis poderia representar um avanço na construção do modelo. Desse modo, constata-se ainda que, na literatura pertinente de ECCB, outros fatores poderiam ser agregados ao modelo, como credibilidade da mensagem, confusão do consumidor, impacto de preço, conveniência, entre outros. Esses fatores agregados poderiam explicar melhor a ECCB.

No que se refere às implicações gerenciais, observa-se que os cosméticos são produtos constituídos por substâncias naturais ou sintéticas, de uso externo nas diversas partes do corpo, com o objetivo principal de lavá-las, perfumá-las, alterar sua aparência, corrigir odores corporais e proteger o corpo ou mantê-lo em bom estado. Esse mercado, no Brasil, é regulamentado e fiscalizado pela Agência Nacional de Vigilância Sanitária (Anvisa).

O mercado mundial de cosméticos, no final da década passada, atingiu UU\$ 269,9 bilhões, representando um crescimento médio de 5,6\% ao ano em relação ao faturamento obtido no início da mesma década (ASSOCIAÇÃO BRASILEIRA DE DESENVOLVIMENTO INDUSTRIAL, 2012). Segundo dados divulgados pela Associação Brasileira da Indústria de Higiene Pessoal, Perfumaria e Cosméticos (ABIHPEC), o Brasil apresentou um faturamento de 27,3 bilhões de reais em 2011, havendo um crescimento de mais de $\mathrm{R} \$ 22$ bilhões, se for realizada uma comparação com as receitas obtidas 15 anos mais cedo (4,9 bilhões). Ampliando as informações para o contexto de exportação, o mercado de cosmético brasileiro teve um crescimento acumulado de $165 \%$ entre 2005 e 2010, atingindo, no ano 2009, o faturamento de US\$ 587,5 milhões e tendo seus produtos comercializados em 135 diferentes países (ASSOCIAÇÃO BRASILEIRA DA INDÚSTRIA DE HIGIENE PESSOAL, PERFUMARIA E COSMÉTICOS, 2012).

Tendências destacadas por estudos da área indicam caminhos promissores para produtos naturais, como extrato de flores, frutas e sementes, bem como de fabricação orgânica, resultantes de uma preocupação cada vez maior com ingredientes sintéticos que possam agredir a pele das usuárias e usuários. É destacado, ainda, um crescimento para o setor nos mercados emergentes (Brasil, China e Rússia), já que estes apresentam uma evolução muito superior à verificada nos demais países durante o período de 2000 a 2010 (ASSOCIAÇÃO BRASILEIRA DE DESENVOLVIMENTO INDUSTRIAL, 2012). Com base nessas evidências, constata-se que o consumo de cosméticos constitui um tema evidente, especialmente 
pela diversidade de produtos existentes, pelo crescimento da indústria e pelo aumento do número de lojas no varejo. E esses fatores enfatizam a utilização cada vez mais frequente de técnicas para analisar as formas de consumo desses produtos, que possam auxiliar as ferramentas de marketing nesse segmento.

\section{REFERÊNCIAS}

ANDERSON, J. C.; GERBING, D. W. Structural equation modeling in practice: a review and recommended two-step approach. Psychological Bulletin, v. 103, n. 3, p. 411-423, 1988.

ARNAU, R. C.; THOMPSON, B. Second-order confirmatory factor analysis of the WAIS-III, v. 3, n. 7, p. 237-246, 2000.

ASSOCIAÇÃO BRASILEIRA DA INDÚSTRIA DE HIGIENE PESSOAL, PERFUMARIA E COSMÉTICOS. Relatório anual, 2012.

ASSOCIAÇÃO BRASILEIRA DE DESENVOLVIMENTO INDUSTRIAL. Relatório de acompanhamento setorial de cosméticos, v. 2, 2012.

BAGOZZI, R. P.; HEATHERTON, T. F. A general approach to representing multifaceted personality constructs: application to state self-esteem. Structural Equation Modeling, v. 1, n. 1, p. 35-67, 1994.

BENTLER, P.; CHOU, C. Practical issues in structural equation modeling. Sociological Methods and Research, v. 16, n. 1, p. 78-117, 1987.

BYRNE, B. M. Structural Equation Modeling: Basic Concepts, Application, and Programming. Mahwah, NJ: Lawrence Erlbaum Associates, 1998.

CARLSON, L.; KANGUN, N.; GROVE, S. J. A. Content Analysis of Environmental Advertising Claims. In: CONFERENCE OF THE AMERICAN ACADEMY OF ADVERTISING, 1., 1992, San Antonio. Anais... San Antonio: TX, 1992.

CATELL, R. B. Questionnaire psychometric properties: test evaluation and improvement. San Fransisco: Jossey-Bass, 1973.

COFFMAN, D. L.; MacCALLUM, R. C. Using parcels to convert path analysis models into latent variable models. Multivariate Behavioral Research, v. 40, n. 2, p. 235-259, 2005. 
FORNELL, C.; LARCKER, D. Evaluating structural equation models with unobservable variables and measurements error. Journal of Marketing Research, v. 17, n. 1, p. 39-50, 1982.

GERBING, D. W.; HAMILTON, J. G.; FREEMAN, E. B. A large-scale secondorder structural equation model of the influence of management participation on organizational planning benefits. Journal of Management, n. 20, v. 4, p. 859-885, 1994.

GURAU, C.; RANCHHOD, A. International Green Marketing: A Comparative Study of British an Romanian Firms. International Marketing Review, v. 22, n. 5, p. 547-561, 2005.

HAANÄÄ, L. Consumers' green commitment: indication of a postmodern lifestyle? International Journal of Consumer Studies, v. 31, p. 478-486, 2007.

HAIR, J. F. et al. Analisis multivariante. 7. ed. Upper Saddle River, NJ: Prentice-Hall, 2009.

JARVIS, C. B.; MACKENZIE, S. B.; PODSAKOFF, P. M. A critical review of construct indicators and measurement model misspecification in marketing and consumer research. Journal of Consumer Research, n. 30, p. 199-218, 2003.

KEESLING, G.; KAYNAMA, S. An Exploratory Investigation of the Ecologically Conscious Consumer's Efforts to Control Water Contamination: Lawn Care and the Use of Nitrogen Fertilizers and Pesticides. Journal of Marketing Theory and Practice, v. 11, n. 1, p. 52-61, 2003.

KIRSHTON, J. M.; WIDAMAN, K. F. Unidimensional versus domain representative parceling of questionnaire items: an empirical example.

Educational and Psychological Measurement, n. 54, p. 757-765, 1994.

KLINE, R. B. Principles and practice of structural equation modeling. 3. ed. New Yorl: The Guilford Press, 2010.

KOUFTEROS, X.; BABBAR, S.; KAIGHOBADI, M. A paradigm for examining second-order factor models employing structural equation modeling. International Journal Production Economics, p. 633-652, 2009.

LILJEBLOM, E.; VAIHEKOSKI, M. Corporate ownership and managerial shorttermism: results from a Finnish study of management perceptions. International Journal of Production Economics, v. 2, n. 117, p. 427-438, 2009. 
MARSH, H. W.; HOCEVAR, D. Application of confirmatory factor analysis of the study of selfconcept: first and higher order factor models and their invariance across groups. Psychological Bulletin, v. 3, n. 97, p. 562-582, 1985.

MITCHELL, A. The Nine American Lifestyles. New York: Macmillan Publishing Co. Inc., 1983.

MULAIK, S. A. et al. Evaluation of goodness-of-fit indices for structural equation model. Psychological Bulletin, v. 105, n. 3, p. 430-445, 1989.

OTTMAN, J. A. Green Marketing. Chicago: NTC Business Books, 1994.

PAÇO, A. M. F.; RAPOSO, M., L. B. Green consumer market segmentation: empirical findings from Portugal. International Journal of Consumer Studies, v. 34, p. 439-436, 2010.

PEARCE, F. The Consumers Are Not So Green. New Scientist, 1990.

RINDSKOPF, D.; ROSE, T. Some theory and applications of confirmatory second-order factor analysis. Multivariate Behavioral Research, v. 1, n. 23, p. 51-67, 1988.

ROBERTS, J. A. Green consumers in the 1990s: profile and implications for advertising. Journal of Business Research, v. 36, n. 3, p. 217-231, 1996.

SAMDAHKL, D. M.; ROBERTSON, R. Social Determinants of Environmental Concern: Specification and Test of the Model. Environment and Behavior, v. 21, n. 1, p. 57-81, 1989.

SCHUMACKER, R. E.; LOMAX, R. G. A beginner's guide to structural equation modeling. New Jersey: Lawrence Erlbaum, 1996.

STERN, P. C.; DIETZ, T.; KALOF, L. Value orientations, gender, and environmental concern. Environment and Behavior, v. 25, n. 5, p. 322-348, 1993.

STISSER, P. A. Deeper Shade of Green. American Demographics, n. 16, p. $24-$ 29, 1994.

STRAUGHAN, R.; ROBERTS, J. A. Environmental segmentation alternatives: a look at green consumer behavior in the new Millennium. Journal of Consumer Marketing, v. 16, n. 6, p. 558-575, 1999.

YIN, R. K. Estudo de caso: planejamento e métodos. 2. ed. Porto Alegre:

Bookman, 2001. 
ZIMMER, M. R.; STAFFORD, T. F.; STAFFORD, M. R. Green issues: dimensions of environmental concern. Journal of Business Research, v. 30, n. 1, p. 63-74, 1994.

\section{Como citar este artigo:}

LADEIRA, Wagner Junior; SANTINI, Fernando Oliveira; ARAUJO, Clecio Falcão. Aplicabilidade da Escala ECCB ao Paradigma da Modelagem de Segunda Ordem: Uma Análise do Consumo Consciente de Cosméticos. RACE, Revista de Administração, Contabilidade e Economia, Joaçaba: Ed. Unoesc, v. 14, n. 2, p. 451-478, maio/ago. 2015. Disponível em: < http://editora.unoesc.edu.br/ index.php/race >. Acesso em: dia/mês/ano.

Ladeira, W. J., Santini, F. O., \& Araujo, C. F. (2015). Aplicabilidade da Escala

$\llbracket$ ECCB ao Paradigma da Modelagem de Segunda Ordem: Uma Análise do

\& Consumo Consciente de Cosméticos. RACE, Revista de Administração, Contabilidade e Economia, 14(2), 451-478. Recuperado em dia/mês/ano, de http://editora.unoesc. edu.br/index.php/race 